Proceedings of the 2011 Winter Simulation Conference

S. Jain, R.R. Creasey, J. Himmelspach, K.P. White, and M. Fu, eds.

\title{
A SIMPLE FRAMEWORK TO SIMULATE THE MOBILITY AND ACTIVITY OF THEME PARK VISITORS
}

\author{
Vladimir Vukadinovic \\ Fabian Dreier \\ Stefan Mangold \\ Disney Research Zurich \\ Clausiusstrasse 47 \\ Zurich, CH-8092, SWITZERLAND
}

\begin{abstract}
Human mobility often needs to be simulated in order to evaluate various designs in transportation and urban planning. Our target application is the design and evaluation of wireless networks and services for new theme park experiences. The performance of some wireless networks, such as mobile ad hoc networks, strongly depends on human mobility. Therefore, we developed a tool named ParkSim that allows us to simulate the mobility of theme park visitors. The tool implements an activity-based mobility model, where the mobility of park visitors is driven by the activities they wish to perform in the park. The tool is calibrated based on GPS tracks collected in an entertainment theme park and validated on a number of metrics that are relevant for the performance of wireless ad hoc networks. ParkSim will be extended to enable the evaluation of new strategies to balance the number of people in different park areas.
\end{abstract}

\section{INTRODUCTION}

Finding ways to model and simulate pedestrian mobility has been the task of transportation and civil engineers for decades. In the area of urban planning, pedestrian mobility patterns are studied in order to design walking facilities, such as sidewalks and underground passages. Civil engineers require knowledge of site-specific mobility in order to design structures such as stadiums, airports, and railway stations. In dense scenarios pedestrian crowds are formed, whose behavior is modeled and simulated in order to study evacuation dynamics. Crowd simulations are also used to animate movements of large groups of characters in movies and video games. In the wireless networking research, pedestrian and vehicular mobility models are used to evaluate the performance of mobile communication systems. These models are typically simplistic compared to those used by transportation and civil engineers.

This paper concerns with human mobility in entertainment theme parks. We present an activitydriven mobility model of park visitors, which we implemented in our ParkSim simulator. The model is calibrated based on the data from theme parks and GPS tracks of more than 600 park visitors. The main purpose of the model is to generate realistic mobility patterns that can be used to study the performance of wireless services offered to park visitors. Personalized location-based services, mobile multiplayer games, park information services, social networking, and multimedia sharing are examples of wireless services that could make a visit to a theme park more interactive and entertaining. The services might rely on software running on existing phones brought by the visitors, or on customized devices (Collins, Muntean, and Mangold 2010). Deploying a wireless infrastructure to support such services in a theme park may create problems that go beyond the obvious deployment and maintenance costs. For example, access points and antennas may be too visible to the guests and, therefore, may interfere with intentions to create certain ambience. For many of the services, continuous connectivity provided by fixed wireless infra- 


\section{Vukadinovic, Dreier, and Mangold}

structures is not needed: The so-called ad hoc mode enables wireless devices to communicate directly when within each other's range. This communication mode is useful when infrastructure-based communication is costly or unavailable. Since devices are mobile (carried by visitors), the ad hoc communication will experience occasional disruptions as links between devices appear and disappear with the mobility. Therefore, it is essential to evaluate the performance of wireless services with realistic mobility models.

Apart from the research on wireless networks, the model can also be used to evaluate various strategies for balancing the number of guests at different locations in a park. Theme parks can often be very crowded, especially during summer vacations and holiday weekends. Long waiting times at popular rides can be the main reason of guest's dissatisfaction. It is desirable to offload crowded areas by giving guests incentives to move to less crowded parts of a park. In the morning hours, for example, visitors tend to cram at the front of the park close to the entrance, while the back of the park is almost empty.

There is already a number of pedestrian mobility models and simulation tools, which are used for research in various fields, including wireless networking. However, they are either simplistic and do not correspond to any specific real-world mobility scenario, or they target some common scenarios, such as urban working-day mobility. For practical applications, models must target specific mobility scenarios, even if this limits the scope of their applicability. We created a model that targets theme park mobility and captures many features that are specific for theme parks. Nevertheless, the model can easily be adapted to scenarios where pedestrians exhibit similar mobility patterns, such as trade shows, zoos, openair museums, and multi-stage festivals. The level of details captured by the model is decided based on the intended use of the model. Many of the available models focus on micro-mobility (walking behavior) and lack proper representation of macro-mobility (choice of destinations driven by person's activity schedule) or vice versa. Our model includes both macro- and micro-mobility. It is still computationally simple enough to simulate the mobility of tens of thousands of people in real-time. We calibrated and validated the ParkSim model based on real-world data from theme parks. The outputs of the simulator are synthetic mobility tracks of park visitors. The tracks contain a sequence of timestamps and corresponding geolocations of a guest in a specified time-resolution.

The remainder of this paper is organized as follows: Section 2 provides an overview of related work. Section 3 describes spatial representations of theme parks in the ParkSim simulator. The mobility model of park visitors is described in Section 4. Section 5 explains how we calibrated various parameters of the model. The model is validated against GPS tracks in Section 6. Section 7 concludes the paper.

\section{RELATED WORK}

It is impossible to capture all details of human mobility in a model. Therefore, models typically focus on aspects that are considered relevant for their intended use (e.g., research in the areas of transportation, urban planning, social sciences, or wireless networking). According to Hoogendoorn, Bovy, and Daamen (2001), human mobility can be described at three levels: strategic, tactical, and operational. Mobility at the strategic level is often referred to as macro-mobility. The tactical and operational levels constitute micro-mobility.

- At the strategic level, people decide on their destinations based on activities that they want to perform, as well as the order in which the activities are performed. It is extremely difficult to model human behavior at this level. Existing models focus on particular mobility scenarios (e.g., working day mobility, airport mobility). They are often empirical and based on observations and surveys.

- At the tactical level, people decide which routes to take between destinations. In reality, route choices are often results of some complex utility maximization which includes many route parameters (travel time, distance, attractiveness, safety, etc). Yet most route-choice models used in simulations, including ours, are based on simple shortest path algorithms.

- At the operational level, people chose their walking speed and direction to avoid collisions with obstacles and other pedestrians. Mobility models used in the research of wireless networks typi- 


\section{Vukadinovic, Dreier, and Mangold}

cally assume free, unobstructed flow of people. However, in ad hoc networks, interaction among pedestrians may affect the rate and the duration of contact opportunities used for data transfer.

Here we provide a brief overview of existing mobility models and simulation tools with a special focus on those that are commonly used in the wireless networking research.

The Random Waypoint (RWP) (Johnson and Maltz 1996) is a popular model that describes mobility patterns of independent, non-interacting nodes in an open area. Each node moves along a zigzag line from one waypoint to the next. RWP model is elementary and it is easy to argue about the paths being unnatural. It owns its popularity to simplicity and mathematical tractability. The RWP has been used as a basis for many other models. Based on empirical observations Rhee et al. (2008) propose Levy Walk model, which assumes power-law distributions of trip lengths between waypoints and pause times in the waypoints. Constrained Random Waypoint models, such as the one proposed in Tian et al. (2002), include geographical restrictions - nodes are restricted to travel between waypoints using pathways. A survey of random walk models is provided in Bai and Helmy (2006). The macro-mobility in these models is simplistic; destinations and their visit order are chosen randomly without any strategic planning. This type of mobility hardly represents any realistic scenario. On the operation level, free unobstructed movement of people is typically assumed. The effect of these assumptions in the performance evaluation of wireless ad hoc networks has been studied in Bai, Sadagopan, and Helmy (2003).

Similar to our model, some models have been derived based on observed mobility and geographical data. For example, the Weighted Waypoint model (Hsu et al. 2005) defines a set of destinations, such as restaurants and classrooms on a university campus. Based on empirical distributions of pause times at each destination and transition probabilities between the destinations, the model constructs a Markov chain of nodes' movements. The UDel mobility model (Kim, Sridhara, and Bohacek 2009) aims to capture a typical day-cycle of a working person based on data collected by the US Bureau of Labor Statistics. In the model, the mobility of people is driven by activities performed on a realistic city map: schedules and durations of activities are calibrated based on the data. Similarly, the model in Zheng, Hing, and Liu (2006) uses data from the US National Household Travel Survey. A drawback of using statistics aggregated from various locations is that models become rather generic. Other examples of empirical models are the Working Day Movement model implemented in the ONE simulator (Ekman et al. 2008) and the model implemented in the CanuMobiSim simulator (Stepanov, Marron, and Rothermel 2005). Most empirical models employ strategic destination planning based on activity scheduling. Scheduled activities are mapped to destinations where they can be performed. Activity-based user modeling belongs to the mature field of travel demand modeling in transportation research. Surveys of activity-driven approaches are provided in (Timmermans 2001; Konig-Ries, Klein, and Breyer 2006).

Elaborate efforts to capture human walking behavior on the operation level have been made in the fields of urban and transportation planning and traffic engineering. The social force model (SFM) described in Helbing and Molnar (1995) assumes that each pedestrian is driven by two types of forces: social and physical. The social forces reflect the intentions of pedestrians not to collide with other people and obstacles; in response to these forces pedestrians accelerate or decelerate. Several commercial multiagent simulators use the SFM to generate mobility; the most notable are VISSIM (2011), Legion Studio (2011), and SimWalk (2011). Besides the SFM, cellular automata models (CAM) provide convenient way to capture human walking behavior (Blue and Adler 2000). In the CAM models, walking space is divided into small cells, which can generate potential fields that represent the local effect of obstacles and other pedestrians on walking direction and speed. PedGo (PedGo 2011) is an example of a simulator based on the CAM. VISSIM, Legion Studio, SimWalk, and PedGo are often used in urban and traffic planning to design public spaces such as airports, subway stations, and sport stadiums. They aim to capture speeddistance relations that emerge when pedestrians navigate obstacles and other pedestrians. These relations are responsible for the formation of pedestrian crowds - capturing them is of paramount importance in emergency evacuation scenarios. These models are often called microscopic since they focus on walking behavior rather than on strategic macro-mobility. Therefore, defining large-scale mobility scenarios using 
these tools requires significant efforts from users. Furthermore, they are often computationally intensive, which limits the number of pedestrians that can be simulated.

\section{PARK REPRESENTATION}

The first step to simulate the mobility of theme park visitors is to create a representation of park's spatial layout in the simulator. ParkSim does not implement its own layout editor where a theme park could be drawn. Instead, the park layout is specified in the OpenStreetMap (OSM) format (OpenStreetMap 2011). Therefore, any OSM editor can be used. An advantage of using the OSM is that large parts of theme parks are already mapped in details, as shown in Figure 1. The OSM maps are easily parsed by the simulator because they use XML syntax. We distinguish between two types of areas in parks: walking areas and activity areas. Visitors use walking areas to move between activity areas. The outlines of the areas need to be specified in the OSM map editor before the park map is parsed by the simulator.

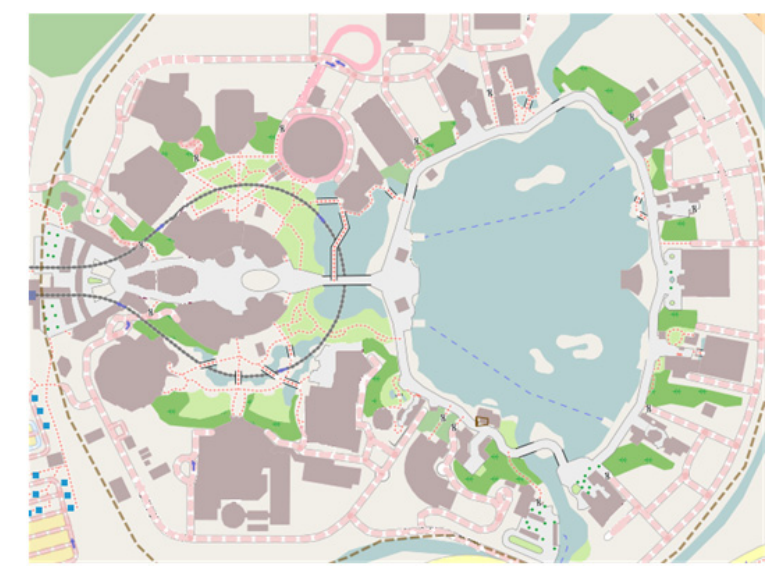

Figure 1: An example of a theme park layout in the OpenStreetMap. Activity and walking areas need to be specified in the OSM editor. Most walking areas are already in the map.

\subsection{Walking Areas}

Most walking areas are already specified as such in OSM maps. We distinguish between two types of walking areas: walkways and plazas. In the OSM, a walkway is represented by a series of waypoints. In ParkSim, this series is split up into segments where each segment contains only two consecutive waypoints. The shape of segment is then computed as a rectangle whose height is equal to the distance between the waypoints and the width is set according to the assumed width of the walkway. Figure. 2 (left) illustrates the geometry of a walkway. A plaza is represented as a polygon with an arbitrary number of edges. A number of connection points located at the edges of the polygon connect the plaza to the adjoining walkways, as shown in Figure 2 (right). For simplicity, we assume that guests use the shortest path when they move between two connection points (i.e. two adjoining walkways) across a plaza. One drawback of this is that the shortest path may fall outside of a concave polygon. This can be avoided by splitting up such plaza into multiple smaller convex plazas.

\subsection{Activity Areas}

Activity areas are locations where park guests perform typical park-related activities. We distinguish between four different types of activity areas: attractions, rides, restaurants, and event areas. The activity areas need to be specified as polygons in the OSM editor before being parsed by the simulator. The characteristics and parameters that can be configured for the activity areas are:

- An attraction is an indoor or outdoor location that guests can visit. An attraction does not have an entrance policy since it is usually able to accommodate all its visitors except when the park is ex- 
Vukadinovic, Dreier, and Mangold
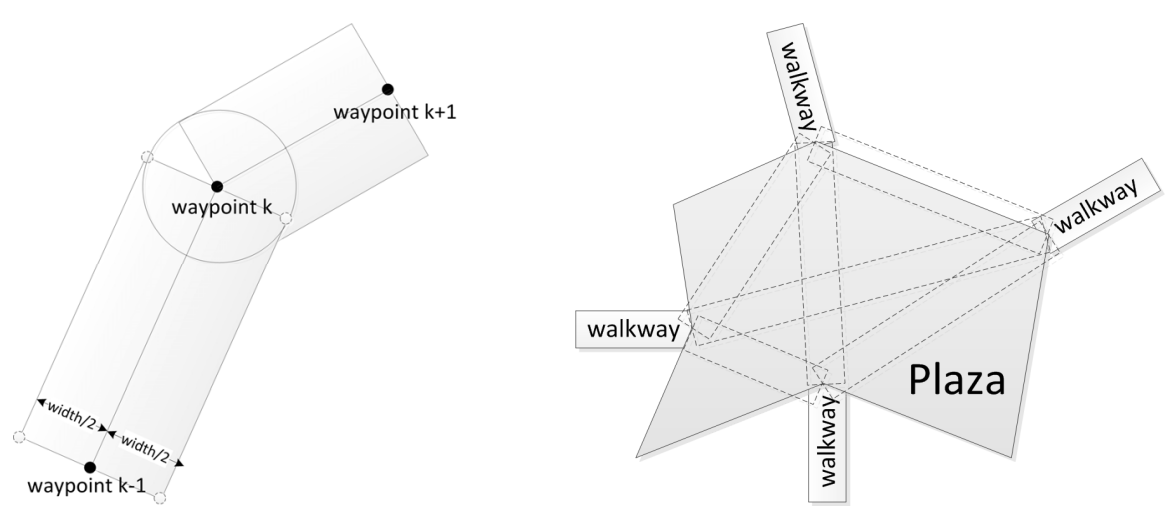

Figure 2: Left: Geometric representations of a walkway. Right: A plaza with adjoining walkways.

tremely crowded. There is a single entrance/exit point to each attraction from adjoining walking areas. The probability distribution of visit durations and mobility model by which guests move inside an attraction can be specified in the simulator.

- A ride is a special type of an attraction whose capacity is limited to the extent that an entrance policy must be specified. There is a queuing space at the entrance to the ride where guests can wait to enter if the ride is currently full. A reservation scheme described in Section 4.1 allows guests to take a so-called fastpass ticket for the ride to avoid long queues. The capacity and ride's duration can be specified in the simulator.

- A restaurant is an eating area, which has a specified capacity, but no queue at the entrance. When in a restaurant, guests are static. The capacity and the probability distribution of visit durations can be specified in the simulator.

- An event area is an area where visitors gather to watch some popular park events, such as street performances and fireworks. An event area encompasses some of the walking areas on which guests may stand to watch the event. The starting time, duration, and popularity of an event can be specified in the simulator.

\section{MOBILITY MODEL}

Guests arrive to the park entrance according to an empirical arrival rate distribution. The arrival rate depends on the time of the day. The total number of arrivals is a parameter of choice (parks tend to be more crowded on weekends and holidays). Once they pass the entrance gate, their mobility is driven by the model implemented in the ParkSim simulator. The model describes mobility at two levels: Macro mobility determines how guests select activity areas to visit and how they prioritize between different activities. Micro mobility determines how they move between and inside activity areas and how they avoid colliding with each other in walking areas and in queue lines. The total time that a guest spends in the park is drawn from an empirical visit time distribution. At the end of his visit, the guest walks towards the exit.

\subsection{Macro Mobility}

A guest can be in one of the following three states: walking (guest is moving in a walking area), visiting (guest is visiting an activity area), or queuing (guest is waiting to enter a ride). Initially, a guest is in the walking state and appears at the park entrance. He then chooses to visit one of the activity areas that are specified as possible initial destinations. The initial destinations are typically located close to the park entrance. The guest walks towards the initial destination (activity area) using the shortest path provided by the walking areas.

At the destination, the guest can either i) enter the activity area if the area is not full; his state changes to visiting, ii) if the activity area is a ride, the guest can join the queue; his state changes to queuing, iii) decide to visit the area at some later time; his state remains unchanged as he walks toward the next desti- 
nation. When a guest enters an activity area, he randomly chooses visit duration from an area-dependent visit duration distribution. In case of rides and events, the visit duration is deterministic. While inside an activity area, the guest moves according to an area-specific micro-mobility model. At the end of the visit, the guest chooses his next activity area based an activity matrix. The activity matrix contains probabilities with which guests chose to visit other attractions and rides in the park, given the last visited attraction or ride. The probabilities are derived from GPS tracks of park visitors, which are described in Section 5.1. Currently, the activity matrix does not account for possible differences between guests of different age and/or sex. Visits to the restaurants and event areas are not driven by the activity matrix, but by guests' hunger and by a timetable of park events, respectively. When a guest selects his next destination, his state changes to walking. The state transition diagram is shown in Figure 3 (left).
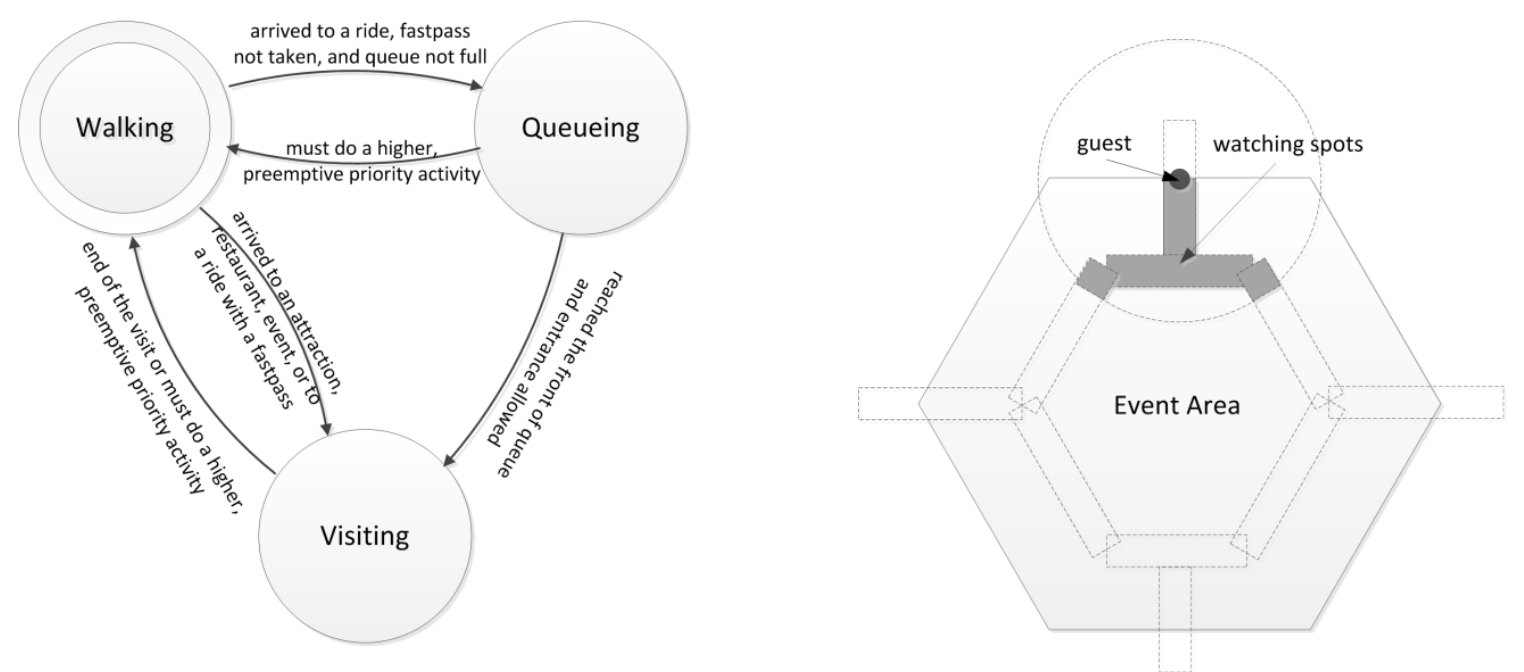

Figure 3: Left: A guest can be in one of the three states: walking, queuing, or visiting. The diagram shows transitions between the states. Right: An event area with an indication of possible watching spots.

Different activity areas (attractions, rides, restaurants, event areas) have different priorities. It may happen that, for example, a guest interrupts his visit to an attraction in order to attend an event. Therefore, an event may have a preemptive priority over some other activities. A guest may, for example, decide to visit a restaurant, but he will do so after his visit to an attraction is finished. Hence, a restaurant visit has a non-preemptive priority over attractions. Priorities of different activities are summarized in Table 1. Note that different events may have different priorities. In the following, we provide more detailed descriptions of how guests queue to enter rides, and how they visit restaurants and events.

Table 1: Different types of activities are scheduled and prioritized.

\begin{tabular}{|l|l|l|}
\hline Activity area & Visits driven by & Priority \\
\hline \hline attraction, ride & activity matrix & low non-preemptive \\
\hline Restaurant & guest's hunger & medium non-preemptive \\
\hline Event & event timetable & medium non-preemptive or high preemptive \\
\hline ride (after taking a fastpass) & time on the fastpass & high preemptive \\
\hline
\end{tabular}

\subsubsection{Fastpass Model}

In some theme parks, a guest who does not want to wait in a queue to enter a ride may take a so-called fastpass. The fastpass specifies a 30-minute slot in which the owner has to return to the entrance of the 
ride in order to enter without queuing. In our simulator, a guest decides to join the queue or to take a fastpass depending on the estimated waiting time $\mathrm{T}_{\mathrm{W}}$, which is calculated based on the number of guests in the queue, the capacity of the ride, and the ride's duration. In theme parks, estimated waiting times are often displayed on screens. Since we lack empirical evidence, we assume that the probability of taking a fastpass $\mathrm{P}_{\mathrm{FP}}$ increases linearly with $\mathrm{T}_{\mathrm{W}}$, and it is equal to one for $\mathrm{T}_{\mathrm{W}}>90 \mathrm{~min}$. The guest joins the queue with the probability $1-\mathrm{P}_{\mathrm{FP}}$. The starting time of the fastpass validity is calculated as the current time plus the estimated waiting time, rounded up to the next full or half-to-full hour. A guest can visit other activity areas until the validity of the fastpass starts. However, he gives a high priority to the ride for which he holds a fastpass: if he is visiting another activity area, he will estimate the time to walk back to the ride and leave the current area early enough to use his fastpass. A guest can hold only one fastpass at a time.

\subsubsection{Restaurant Visit Model}

The probability that the next activity area visited by a guest is a restaurant increases linearly with the time spent in the park. Six hours after entering a park, the guest will eat at least once. The probability of choosing a particular restaurant is inversely proportional to the walking distance to the restaurant. In addition to the distance, restaurant popularity could be accounted for. However, due to the lack of empirical data, we currently assume that all restaurants are equally popular.

\subsubsection{Event Visit Model}

An event area is defined in the OSM map as a polygon that encompasses some of the park's walking areas. The walking areas inside the event area can be used by guests to select a spot to watch the event (e.g., firework, street performance). When a guest arrives to the event area, he will select a random spot in the walking areas that are within a certain radius, as shown in Figure 3 (right). This avoids overcrowding of the walking areas when only few walkways enter the event area. An event has a starting time, duration, spot selection radius, popularity, and priority as parameters. The popularity is the probability that a random guest in the park will attend the event. An event may have a medium non-preemptive priority (e.g., a street parade) or high preemptive priority (e.g., a park evacuation).

\subsection{Micro Mobility}

Micro-mobility determines how guests select routes between activity areas, how they avoid colliding with each other in walking areas, and how they move inside activity areas and queues.

\subsubsection{Routing}

ParkSim uses the Dijkstra algorithm to calculate shortest paths between activity areas. The algorithm computes an ordered list of walkways that determines waypoints for walking. A guest walks straight towards the next waypoint if not disturbed by other guests; otherwise the direction of his movement is determined by a collision avoidance algorithm. To avoid cases where all guests walk in the middle of the walkway, a random offset is added to the waypoint so that the guest actually moves towards a randomly selected point on the ending edge of the current segment. Target walking speeds of guests are drawn from a specified speed distribution. The actual walking speeds depend on the crowdedness of a park.

\subsubsection{Collision Avoidance}

Simulated guests try to avoid colliding with each other while walking. The guests are represented by circles of a specified radius. Each guest has a rectangular field of view, as shown in Figure 4 (left). The figure illustrates a scenario where two guests would collide after three simulation timeslots/steps if their walking directions remain unchanged. The collision avoidance algorithm extrapolates the trajectories of 
all persons within the field of view of a guest in order to predict impeding collisions. If one or more collisions are predicted, the algorithm changes the direction and/or the speed of the guest to avoid the collision(s). The algorithm will first try to change the directions without changing the speed. It this is not possible, for example because the guest would have to step outside of the walking area, the speed is changed. We omit the details of the algorithm for brevity. The algorithm is executed at each time step for each guest independently. It is a best-effort algorithm, which does not ensure a completely collision free movement, but it captures the effect of crowded walking areas on the speeds of the guests.

\subsubsection{Queuing Behavior}

A queue line is modeled as a grid of cells with specified width and length. Each cell provides space for one guest, as shown in Figure 4 (right). When entering the queue, a guest selects a free cell that is closest to the entrance; ties are broken randomly. The guest moves forward whenever some of the guests in front of him are allowed to enter the ride. Since the number of cells is limited, it may happen that an arriving guest finds all cells occupied. In such cases, he selects another activity area to visit, according to the activity matrix. Note that a guest who decides to take a fastpass does not enter the queue.

\subsubsection{Intra-Area Mobility}

When they are inside activity areas, the mobility of guests is different from the mobility in walking areas. Currently, there are two intra-area mobility models in the ParkSim: Random Waypoint and Random Sitpoint models. In the popular Random Waypoint model (Johnson and Maltz 1996), a guest moves from one random waypoint to the other along a zigzag line that connects them. This model is used for some attractions and rides. In the Random Sitpoint model, a guest moves towards a random point inside an activity area and remains there until the end of his visit. This model is used for restaurants, event areas, and some attractions and rides.

\section{MODEL CALIBRATION}

Three theme parks have been modeled in the ParkSim so far: two located in the USA and one in France. We use one of the theme parks in the USA as an example to describe how we calibrated and validated the model. The layout of the park is shown in Figure 1. The ParkSim model of the park contains 22 attraction areas, six rides, 12 restaurants, and an event area centered around the lake. During the calibration, various parameters of the model, described in Sections 3 and 4, are set based on data collected in the park. The data comes from two sources: long-term visit statistics collected by the park management and GPS tracks of guests collected in a measurement campaign. The hourly distribution of guests' arrivals to the park, schedules and durations of park events (e.g., fireworks on the lake), and ride durations are obtained from the park management. Routing probabilities between the attractions/rides and distributions of visit dura-
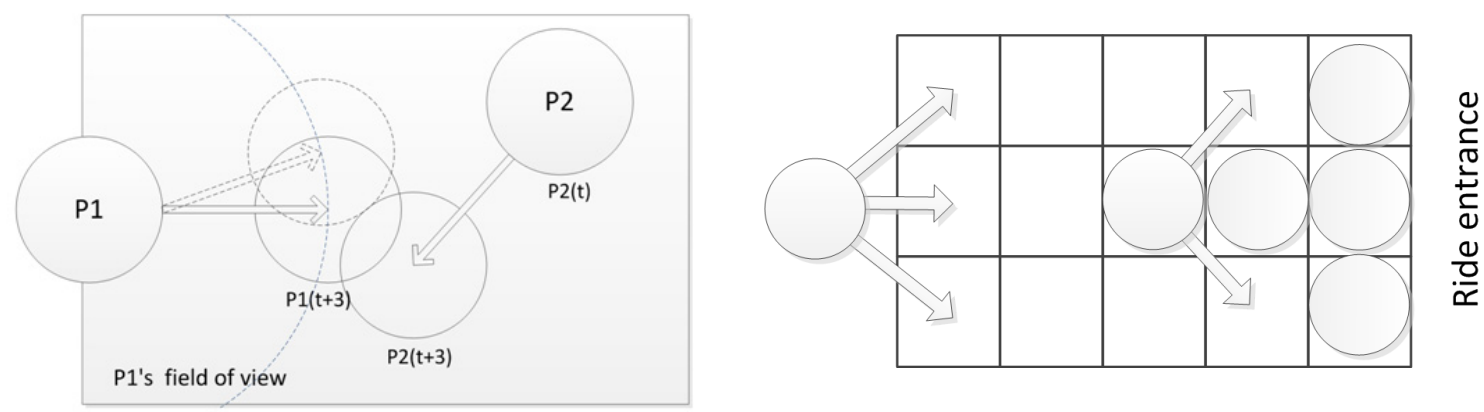

Figure 4: Left: An example of a potential collision that is avoided by changing the direction of movement. Right: An example of a queue line structure. 
tions for the attractions are obtained from the GPS tracks, which are described later in this section. Most of the data is confidential and, therefore, not disclosed in this paper. Some of the model parameters are set arbitrary due to the lack of relevant data (e.g., visit durations for the restaurants).

\subsection{GPS Tracks of Park Visitors}

GPS tracks are used both for calibration and validation of the model. The tracks were collected during a behavioral research study. Over the course of five days, 200 smartphones were distributed each day to a total of 910 randomly-selected visitors. In case of groups/families only one of the members was selected. The phones ran an application that logged their GPS locations on average every two minutes when the satellite signals were available. The tracks contain gaps, which correspond to the periods when visitors were indoors (e.g., in buildings where the GPS signal is not available). During such periods, we assume that the visitor was located somewhere close to the building where the last waypoint was recorded. In addition to geo-coordinates, GPS accuracy (reported by GPS itself) was logged. We discarded waypoints whose accuracy was worse than 25 meters. We interpolated the movement of visitors between the remaining waypoints assuming straight-line movement. We discarded the tracks shorter than five hours and/or containing less than 50 waypoints. The model calibration and validation are based on the remaining 670 tracks. The activity matrix with routing probabilities, visit durations, and validation statistics (described in the following section) are extracted from the GPS tracks with the help of Matlab scripts.

\section{MODEL VALIDATION}

Assume that park visitors are equipped with portable radio devices. We say that two devices are in contact if they are within each other's radio range. We assume that the range is fixed and equal to 50 meters. The model validation focuses on several contact-related metrics that are highly relevant for the performance of data forwarding algorithms in wireless ad hoc networks: inter-contact times, contact durations, number of neighbors, and mean square displacement. The statistical distributions of the metrics are calculated both based on the GPS tracks and synthetic tracks produced by the ParkSim. The results are compared against each other. In case of a good agreement, we consider the model valid. Our validation aims to check if the model captures mobility aspects that are relevant for the performance evaluation of wireless ad hoc networks. Validation results do not imply that our model captures all details of park mobility.

\subsection{Contact Durations and Number of Neighbors}

Contact duration is the time two devices/visitors remain in contact. Many short contacts arise when park visitors are bypassing each other. Longer contacts occur when visitors are static and collocated (e.g., while they are waiting in queues or sitting in restaurants). It is therefore not surprising that the distribution of contact durations obtained from the GPS tracks, which is shown in Figure 5 (left), appears to be heterogeneous and heavy-tailed, which is indicated by the slope of the tail (note that the y-axis is logarithmic). The results shown in Figure 5 (left) confirm that the CCDFs of contact durations observed in the two sets of mobility tracks are similar. Slight mismatch is due to our simplifying assumption related to gaps is GPS tracks: We assume that during the gaps visitors remain static, which leads to longer contacts.

Devices with many neighbors (simultaneous contacts) are often desirable as next-hop relays. The number of neighbors of a device changes as visitors move. The average number of neighbors over time indicates the importance of the device for the connectivity of the network. In some data forwarding schemes, devices that are highly central due to many neighbors consume more energy on relaying and, therefore, drain their battery faster. The CCDF of the average number of neighbors is shown in Figure 5 (right). Fast decaying tails indicate that there are relatively few devices that are much better connected than others. The decay is well captured in the ParkSim tracks. 

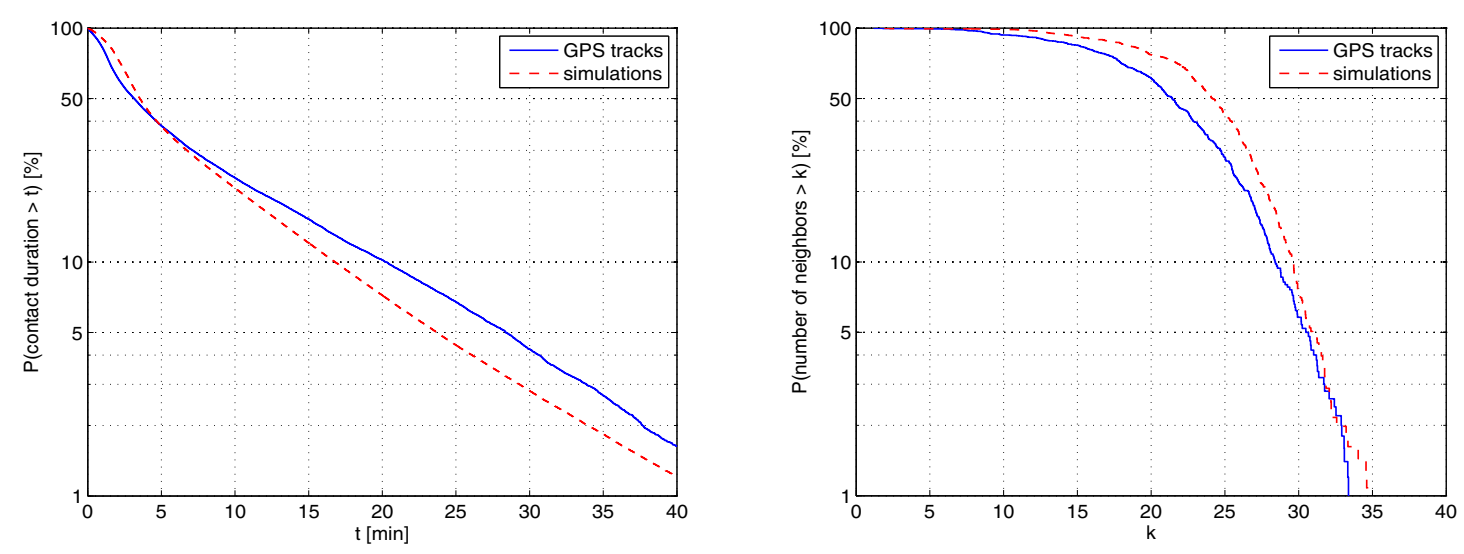

Figure 5: Left: CCDF of contact durations. Right: CCDF of the average number of neighbors over time.

\subsection{Inter-Contact Times}

Inter-contact time (ICT) is the time elapsed between starts of two successive contacts of the same pair of devices/visitors. In multi-hop networks, where messages are relayed from one device to another until they reach the destination, the distribution of ICTs can be used to derive the residual time until a new contact occurs and, therefore, the time until any of the relays encounters the destination of the message. Hence, the distribution of ICTs is closely related to the end-to-end delay of data forwarding algorithms - longer ICTs lead to longer delays. ICTs have been studied extensively in the literature. Results in (Karagiannis, Le Boudec, and Vojnovic 2007) indicate that the tail distribution of ICTs exhibits a dichotomy-it follows power-law decay up to some characteristic time, beyond which the decay is exponential. In Figure 6 (left) we confirm the same tendency in our GPS tracks. The figure shows the complementary cumulative distribution function (CCDF) of ICTs aggregated over all pairs of visitors that encountered each other at least twice. The shape of the curve indicates that the tail indeed follows a power low decay with slope $\sim 0.25$ up to a characteristic time of $\sim 30$ minutes. The fast drop beyond the characteristic time indicates exponentially decaying tail. The same tendency is observed in mobility tracks produced by the ParkSim.

\subsection{Mean Square Displacement}

Displacement measures how far away a visitor is from its starting position after some time $t$. Let $\mathrm{P}_{\tau} \in \mathbb{R}^{2}$ be the position of a visitor at time $\tau$ (e.g., in an x-y coordinate system). Mean square displacement after time $t$ is given by $\operatorname{MSD}(\mathrm{t}) \triangleq \mathbb{E}\left\{\left\|\mathrm{P}_{\tau+\mathrm{t}}-\mathrm{P}_{\tau}\right\|^{2}\right\}$. Hence, it is the second moment of the displacement $\left\|\mathrm{P}_{\tau+\mathrm{t}}-\mathrm{P}_{\tau}\right\|$ between its current position $\mathrm{P}_{\tau+\mathrm{t}}$ and its starting position $\mathrm{P}_{\tau}$. We calculated the MSD(t) of each park visitor by varying $\tau$ from zero to $T-t$, where $T$ is the length of his mobility track. MSD(t) increases with time $t$, such that $\operatorname{MSD}(t) \sim t^{\gamma}$. The exponent $\gamma$ indicates the speed of diffusion. For Brownian motion the MSD increases linearly with time $(\gamma=1)$. When $\gamma>1$, the mobility is superdiffusive. For example, for a visitor walking on a straight line $\operatorname{MSD}(t) \sim \mathrm{t}^{2}$. Visitors whose mobility exhibits stronger diffusion will cover larger area compared to nodes with weaker diffusion. As a consequence, they will encounter more visitors with devices that can serve as data relays. Kim, Lee, and Eun (2010) show that the speed of diffusion makes huge impact on the performance of data forwarding algorithms. We calculated the MSD from GPS tracks to find out about the diffusive behavior of park visitors. In Figure 6 (right), we show the average $\operatorname{MSD}(\mathrm{t})$ for 500 tracks on a $\log$-log scale. The slope of the curve $\gamma>1$ indicates that visitors' mobility exhibits superdiffusive behavior over a few tens of minutes. Since visitors move in a confined space and return to the park gate at the end of their visit, the MSD cannot grow indefinitely. The slope of MSD decreases below one after approximately one hour. Over short time intervals, the MSD in the ParkSim tracks is somewhat larger compared to the GPS tracks. This is due to unavailability of GPS 

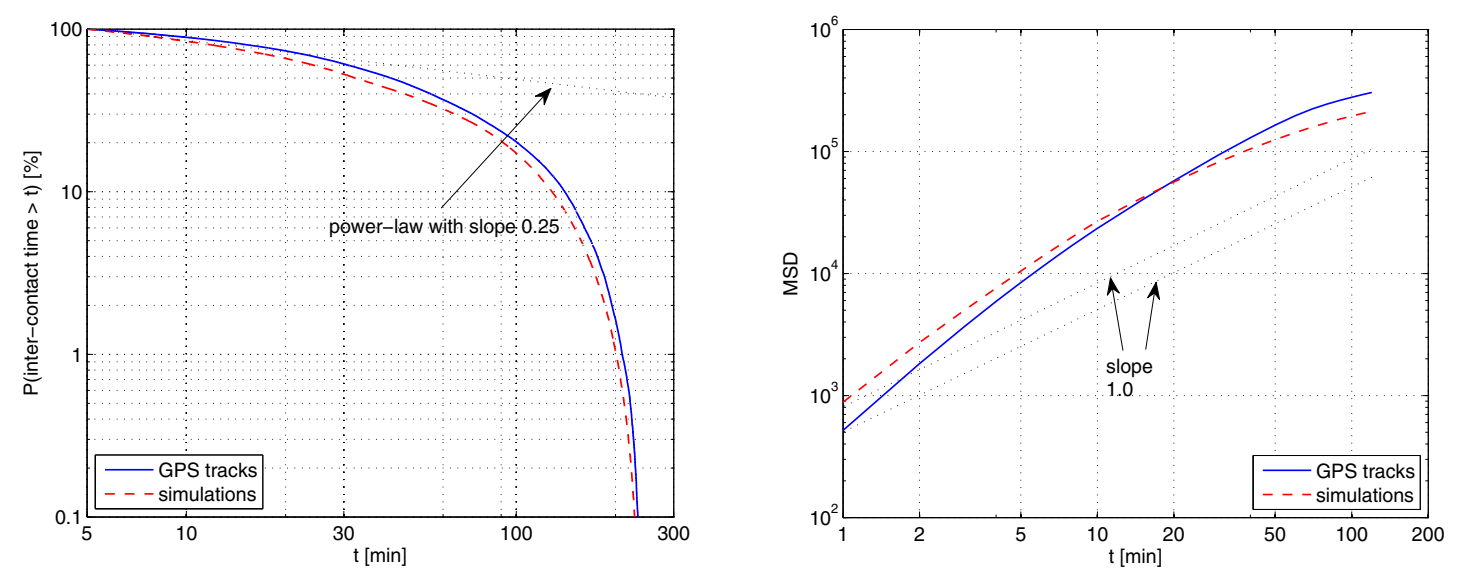

Figure 6: Left: CCDF of inter-contact times aggregated over all pairs of visitors. Right: Mean square displacement averaged over 500 visitors. The mobility of park visitors exhibits superdiffusive behavior over a few tens of minutes as indicated by the slope $\gamma>1$.

signal in indoor locations: In the GPS tracks, we assume that visitors are static while indoors and their MSD is zero during that time, while in the ParkSim tracks they move according to the location-specific indoor mobility models. In spite of this mismatch, the ParkSim tracks capture the superdiffusive behavior observed in the GPS tracks.

\section{CONCLUSIONS}

A framework for realistic simulation of pedestrian mobility in theme parks is presented. The mobility model is implemented in a tool named ParkSim, which will be publicly available as a part of our network simulation toolkit Jemula802 (Jemula802 2011). The model is empirical: it is based on data collected in theme parks. The data provided us with information about activities that visitors perform and helped us construct a macro-mobility model. A simple, computationally light micro-mobility model allows us to simulate a large number of visitors in real time. Synthetic mobility tracks produced by ParkSim are validated against real-world GPS tracks of park visitors. The results indicate that ParkSim is a suitable tool to assist performance evaluations of wireless ad hoc networks. Furthermore, the tool will allow to study the impact of changes in mobility patterns caused by the introductions of new crowd balancing mechanisms.

\section{REFERENCES}

Bai, F., and A. Helmy. 2006. "A Survey of Mobility Models in Wireless Ad Hoc Networks." In Wireless Ad Hoc and Sensor Networks, edited by A. Safwat, 1-30. Springer Verlag.

Bai, F., N. Sadagopan, and A. Helmy. 2003. "IMPORTANT: A framework to systematically analyze the Impact of Mobility on Performance of RouTing protocols for Ad-hoc NeTworks." In Proceedings of the IEEE Infocom, 825-835. San Francisco, USA. Institute of Electrical and Electronics Engineers, Inc.

Blue, V. J., and J. L. Adler. 2000. "Cellular Automata Micro-Simulation of Bi-Directional Pedestrian Flows." Transportation Research Part B 35:135-141. Elsevier.

Collins, K., G. Muntean, and S. Mangold. 2010. "Supporting Mobile Devices with Wireless LAN/MAN in Large Controlled Environments." IEEE Communications Magazine 48:36-43. Institute of Electrical and Electronics Engineers, Inc.

Ekman, F., A. Keranen, J. Karvo, and J. Ott. 2008. "Working Day Movement Model." In Proceedings of the ACM Workshop on Mobility Models for Networking Research, 33-40. Hong Kong, China. Association for Computing Machinery, Inc. 
Helbing, D., and P. Molnar. 1995. "Social Force Model for Pedestrian Dynamics." Physical Review E 51:4282-4286. The American Physical Society.

Hoogendoorn, S. P., P. H. L. Bovy, and W. Daamen. 2001. "Microscopic Pedestrian Wayfinding and Dynamics Modeling." Pedestrian and Evacuation Dynamics, 123-154. Springer Verlag.

Hsu, W.-J., K. Merchant, H.-W. Shu, C.-H. Hsu, and A. Helmy. 2005. "Weighted Waypoint Mobility Model and Its Impact on Ad Hoc Networks." ACM Mobile Computing and Commun. Review 9:5963. Association for Computing Machinery, Inc.

Jemula802. 2011. “Jemula802.” Accessed: March 16, 2011. http://jemula802.origo.ethz.ch.

Johnson, D. B., and D. A. Maltz. 1996. "Dynamic Source Routing in Ad Hoc Wireless Networks." Mobile Computing 353:153-181. Kluwer Academic Publishers.

Karagiannis, T., J.-Y. Le Boudec, and M. Vojnovic. 2007. "Power Law and Exponential Decay of Inter Contact Times Between Mobile Devices." In Proceedings of the ACM Mobicom, 183-194. Montreal, Canada. Association for Computing Machinery, Inc.

Kim, J., V. Sridhara, and S. Bohacek. 2009. "Realistic Mobility Simulation of Urban Mesh Networks." Ad Hoc Networks 7:411-430. Elsevier.

Kim, S., C.-H. Lee, and D.Y. Eun. 2010. "Superdiffusive Behavior of Mobile Nodes and its Impact on Routing Protocol Performance." IEEE Trans. Mobile Computing 9:288-304. Institute of Electrical and Electronics Engineers, Inc.

Konig-Ries, B., M. Klein, and T. Breyer. 2006. "Activity-Based User Modeling in Wireless Networks." Mobile Networks and Applications. 11:267-277. Springer Verlag.

Legion Studio. 2011. "Legion - Pedestrian Simulation Solutions." Accessed: March 16, 2011. http://www.legion.com.

OpenStreetMap. 2011. “OpenStreetMap.” Accessed: March 16, 2011. http://www.openstreetmaps.org.

PedGo. 2011. “TraffGo HT.” Accessed: March 16, 2011. http://www.traffgoht.com/en/pedestrians/products/pedgo/index.html.

Rhee, I., M. Shin, S. Hong, K. Lee, and S. Chong. 2008. "On the Levy-Walk Nature of Human Mobility." In Proceedings of the IEEE Infocom, 630-643. Phoenix, USA. Institute of Electrical and Electronics Engineers, Inc.

SimWalk. 2011. "SimWalk - the simulation software for pedestrian flows." Accessed: March 16, 2011. http://www.simwalk.com.

Stepanov, I., P. Marron, and K. Rothermel. 2005. "Mobility Modeling of Outdoor Scenarios for MANETs." In Proceedings of the 38th Annual Simulation Symposium, 312-322. San Diego, USA. IEEE Computer Society.

Tian, J., J. Hahner, C. Becker, I. Stepanov, and K. Rothermel. 2002. "Graph-Based Mobility Model for Mobile Ad Hoc Network Simulation." In Proc. 35th Annual Simulation Symposium, 337-344. San Diego, USA. IEEE Computer Society.

Timmermans, H. 2001. "Models of Activity Scheduling Behavior." Stadt Region Land 71:33-47. Institut für Stadtbauwesen und Stadtverkehr.

VISSIM. 2011. "PTV AG:VISSIM - Multi-Modal Traffic Flow Modeling.” Accessed: March 16, 2011. http://www.vissim.de.

Zheng, Q., X. Hong, and J. Liu. 2006. “An Agenda Based Mobility Model.” In Proceedings of the 39th Annual Simulation Symposium, 188-195. Huntsville, USA. IEEE Computer Society.

\section{AUTHOR BIOGRAPHIES}

VLADIMIR VUKADINOVIC is a postdoctoral researcher at Disney Research Zurich, Switzerland. He received his Dipl.-Ing. and M.Sc. degrees in electrical engineering and telecommunications from the University of Belgrade, Serbia, in 2000 and 2004, respectively, and his Ph.D. degree in telecommunications from the Royal Institute of Technology, Stockholm, Sweden in 2010. His research interests are focused 
on wireless multimedia networking, delay and disruption tolerant communication, and modeling and simulation of human mobility. His email address is vvuk@disneyresearch.com.

FABIAN DREIER received his M.Sc. degree in computer science from the Swiss Federal Institute of Technology, Zurich, Switzerland in 2011. During his masters studies he was an intern at Disney Research Zurich for more than a year. The main focus of his work is in the field of Wi-Fi network simulation. His e-mail address is fdreier@disneyresearch.com.

STEFAN MANGOLD is a senior research scientist at Disney Research Zurich and lecturer at ETH Zurich, Department of Computer Science. Before joining Disney Research, he worked at Swisscom in research, product and business development, and at Philips Research USA. His research covers many aspects of wireless communication networks and mobile computing, such as wireless protocols and system aspects for IEEE 802.11 Wireless LAN, cognitive radio, and wide area networks. Other research interests include mobile computing, interactive toys, cellular networks, and ontology engineering. He is coauthor of two books, IEEE 802 Wireless Systems: Protocols, Multi-Hop Mesh/Relaying, Performance, and Spectrum Coexistence (Wiley, 2007) and Cognitive Radio and Dynamic Spectrum Access (Wiley, 2009). His e-mail address is stefan@disneyresearch.com. 